Mon. Not. R. Astron. Soc. 000, $1,13(2016) \quad$ Printed 27 October $2016 \quad$ (MN LATEX style file v2.2)

\title{
Revised Geometric Estimates of the North Galactic Pole and the Sun's Height Above the Galactic Midplane
}

\author{
M. T. Karim ${ }^{1 \star}$ and Eric E. Mamajek ${ }^{1,2}$ \\ ${ }^{1}$ Department of Physics \& Astronomy, University of Rochester, Rochester, NY 14627, USA \\ ${ }^{2}$ Current Address: Jet Propulsion Laboratory, California Institute of Technology, 4800 Oak Grove Dr., Pasadena, CA 91109, USA
}

Accepted 2016 October 24. Received 2016 October 22; in original form 2016 September 5

\begin{abstract}
Astronomers are entering an era of $\mu$ as-level astrometry utilizing the 5-decade-old IAU Galactic coordinate system that was only originally defined to $\sim 0^{\circ} .1$ accuracy, and where the dynamical centre of the Galaxy (Sgr $\mathrm{A}^{*}$ ) is located $\sim 0^{\circ} .07$ from the origin. We calculate new independent estimates of the North Galactic Pole (NGP) using recent catalogues of Galactic disc tracer objects such as embedded and open clusters, infrared bubbles, dark clouds, and young massive stars. Using these catalogues, we provide two new estimates of the NGP. Solution 1 is an "unconstrained" NGP determined by the galactic tracer sources, which does not take into account the location of $\operatorname{Sgr} \mathrm{A}^{*}$, and which lies $90^{\circ} .120 \pm 0^{\circ} .029$ from Sgr A*, and Solution 2 is a "constrained" NGP which lies exactly $90^{\circ}$ from Sgr A*. The "unconstrained" NGP has ICRS position: $\alpha_{N G P}=192^{\circ} .729 \pm 0^{\circ} .035, \delta_{N G P}=27^{\circ} .084 \pm 0^{\circ} .023$ and $\theta=$ $122^{\circ} .928 \pm 0^{\circ} .016$. The "constrained" NGP which lies exactly $90^{\circ}$ away from Sgr A* has ICRS position: $\alpha_{N G P}=192^{\circ} .728 \pm 0^{\circ} .010, \delta_{N G P}=26^{\circ} .863 \pm 0^{\circ} .019$ and $\theta=$ $122^{\circ} .928 \pm 0^{\circ} .016$. The difference between the solutions is likely due to the Sun lying above the Galactic midplane. Considering the angular separation between Sgr A* and our unconstrained NGP, and if one adopts the recent estimate of the Galactocentric distance for the Sun of $R_{0}=8.2 \pm 0.1 \mathrm{kpc}$, then we estimate that the Sun lies $z_{\odot} \simeq$ $17 \pm 5 \mathrm{pc}$ above the Galactic midplane. Our value of $z_{\odot}$ is consistent with the true median of 55 previous estimates published over the past century of the Sun's height above the Galactic mid-plane $\left(z_{\odot} \simeq 17 \pm 2 \mathrm{pc}\right)$.
\end{abstract}

Key words: The Galaxy (centre, disc, fundamental parameters, general, kinematics and dynamics, structure)

\section{INTRODUCTION}

"The anticipated improvement in the position of the [Galactic] pole, by a factor of $10 / 1$ or better, is substantial, and is unlikely to be increased for many years" (Blaauw et al. 1960)

While the need for a Galactic Coordinate System (GCS) goes back at least to the time of William Herschel (Herschel 1785), the first standard Galactic coordinate system in common international use was that of Ohlsson (1932). The GCS is defined as a spherical celestial coordinate system with right ascension $\left(\alpha_{P}\right)$ and declination $\left(\delta_{P}\right)$ marking the North Galactic Pole (NGP), and its equator tracing the Galactic plane. In the early 20th century, the zero longitude $\left(\ell=0^{\circ}\right)$ was defined between this pole and the point where the galactic plane intercepted the equinox 1900.0 celestial equator Ohlsson 1932 van Tulder 1942

* Email: mkarim2@u.rochester.edu
Ohlsson et al. 1956). Ohlsson (1932) determined the location of the NGP to be $\alpha_{P}=12^{h} 40^{m}, \delta_{P}=+28^{\circ}$ (B1900.0). van Tulder (1942) later determined a NGP using numerous samples of optical stars, tracing extinction in the Galactic plane, and other indicators, which deviated from Ohlsson's pole by $\sim 1^{\circ} \alpha_{P}=12^{h} 44^{m}\left( \pm 0^{\circ} .3\right), \delta_{P}=+27^{\circ} .5\left( \pm 0^{\circ} .2\right)$ (B1900.0 equinox). By the mid-1950's, it was apparent from H I surveys and the discovery of an obvious dynamical centre to the Galaxy (associated with the radio source Sgr A) that a revision to the van Tulder (1942) GCS was needed.

At the Xth IAU General Assembly in Moscow, IAU Commissions 33 (Stellar Statisics) and 40 (Radio Astronomy) authorized the formation of an IAU sub-commission (33b) to determine a new GCS which principally traced the neutral hydrogen in the Galactic plan\& Summaries of

1 https://www.iau.org/static/resolutions/IAU1958_French.pdf 
the research by IAU Sub-Commission 33b defining the IAU GCS were given in a series of contemporaneous papers by Blaauw et al. (1960, Paper I), Gum et al. (1960, Paper II), Gum \& Pawsey (1960 Paper III), Blaauw (1960, Paper IV) and Oort \& Rougoor (1960, Paper V). Paper I (Blaauw et al. 1960) summarized the sub-commission's research and comprises their proposal of the 1958 revised GCS to the IAU. Using H I survey data by groups at Leiden and Sydney, Paper II (Gum et al. 1960) demonstrated the flatness of the Galactic neutral hydrogen, determined the best-fitting H I Galactic plane, and independently estimated that the Sun was near the plane, within uncertainties (height $z_{\odot}=$ $4 \pm 12$ pc). Further, Paper III (Gum \& Pawsey 1960) argued that the trends in intensity for $\mathrm{HI}$ and radio continuum data were consistent with Sgr A marking the centre of the Galaxy. Paper IV (Blaauw 1960) argued that optical stars (e.g. OB stars, Cepheids, etc.) should not be employed to constrain the NGP due to limited sampling and interstellar extinction, and that these samples appear to be coplanar with the Hi principle plane. Paper V (Oort \& Rougoor 1960) presented further arguments based on optical and radio data for positing Sgr A to mark the dynamical centre of the Galaxy. Based on Blaauw's (1960) argument that the H I plane was a superior means of defining the Galactic plane and NGP (abandoning van Tulder's optical-based methodology), and employing the $\mathrm{HI}$ plane defined by Gum et al. (1960) to define the Galactic plane, and defining the radio source Sgr A as the logical choice of origin (Gum \& Pawsey 1960 Oort \& Rougoor 1960), the commission proposed the IAU GCS to have NGP at $\alpha_{P}^{I A U}=12^{h} 49^{m}$, $\delta_{P}^{I A U}=+27^{\circ} .4$ with position angle $\theta_{P}^{I A U}=123^{\circ}$ (all B1950; Blaauw et al. 1960). The position angle $\theta$ is that of the "the new galactic pole of the great circle passing through Sagittarius $A$ " (Blaauw et al. 1960). On the ICRS, the IAU NGP corresponds to $\alpha_{P}^{I A U}=12^{h} 51^{m} 26^{s} .2755, \delta_{P}^{I A U}=$ $+27^{\circ} 07^{\prime} 41^{\prime \prime} .704$ and $\theta_{P}^{I A U}=122^{\circ} .93191857$ in J2000.0 (Liu et al. 2011b). Furthermore, the position of the Galactic Centre (GC) on the IAU GCS was set to be at $\alpha_{G C}^{I A U}=$ $17^{h} 42^{m} 37^{s}, \delta_{G C}^{I A U}=-28^{\circ} 57^{\prime}$ (B1950; Gum et al. 1960). On the J2000 system this position corresponds to $\alpha_{G C}^{I A U}=$ $17^{h} 45^{m} 37^{s} .224, \delta_{G C}^{I A U}=-28^{\circ} 56^{\prime} 10^{\prime \prime} .23$ (Reid \& Brunthaler 2004).

However, radio surveys in recent decades have been steadily improving our understanding of $\mathrm{Sgr}$ A, resolving the bright nonthermal radio source $\mathrm{Sgr} \mathrm{A}^{*}$ as the true dynamical centre of the Galaxy (e.g. Brown 1982 Reid \& Brunthaler 2004). Reid \& Brunthaler (2004) determined the precise position of Sgr $\mathrm{A}^{*}$ to be $\alpha_{G C}=$ $17^{h} 45^{m} 40^{s} .0409, \delta_{G C}=-29^{\circ} 00^{\prime} 28^{\prime} .118$ (ICRS, epoch 2000.0) with proper motion $\mu_{\alpha}=-3.151 \pm 0.018$ mas yr $^{-1}$ and $\mu_{\delta}=-5.547 \pm 0.026 \mathrm{mas} \mathrm{yr}^{-1}$. Hence, there is an offset of $0^{\circ} .0724$ when comparing the position of the origin of the IAU GCS and Sgr A*. Given the promised improvement in astrometric precision afforded by Gaia (Lindegren et al. 2016), and the ability of the survey catalogue to greatly improve our knowledge of Galactic dynamical parameters (Bland-Hawthorn \& Gerhard 2016), one would prefer that Galactic positions and velocities could be calculated accurately on a natural Galactic coordinate system to at least third significant figure. Hence, it can be argued that the IAU 1958 GCS is no longer an ade- quate representation of the Milky Way's natural orientation.

There has been little published work on refining the Galactic coordinate system since the 1950s IAU effort. Recently Liu et al. (2011a) tried to determine the transformation matrix in the framework of ICRS but could not find a GCS that is connected steadily to the ICRS. Later Liu et al. (2011b) determined estimates of the NGP based on the Two-Micron All-Sky Survey (2MASS) and SPECFIND v2.0 catalogues. More recently Ding et al. (2015) solved for new estimates of the NGP based on points sources in two infrared sky surveys from the AKARI and WISE missions (covering bandpasses between $3.4 \mu \mathrm{m}$ and $90 \mu \mathrm{m}$ ) using the same method as Liu et al. (2011b).

In this paper, we provide independent estimates of the North Galactic Pole using less biased tracers of Galactic structure than have been often used before (e.g. H I, near-IR star counts, etc.). Section 2 discusses the classes of Galactic tracers that we employ and the catalogues of tracers selected for analysis. Section 3 discusses how we calculated the fundamental parameters, i.e. the RA and the Dec of the NGP and the position angle of the NGP with respect to the Galactic Centre at the North Celestial Pole. Lastly, Section 4 compares our estimates of these Galactic parameters to previously published studies, and presents an estimate of the Sun's height above the Galactic midplane based on our revised NGP.

\section{DATA}

\subsection{Categories of Galactic Tracers}

Throughout this study, we consider "Galactic tracers" as classes of astronomical objects that strongly trace the Galactic disc and plane, with obvious examples being $\mathrm{H}$ II regions, embedded clusters, infrared dark clouds (IRDCs), etc. The current IAU definition (Blaauw et al. 1960) depends heavily on the distribution of $\mathrm{HI}$ gas - which has the advantage of tracing mass at large distances, and demonstrates a high degree of flatness in the plane. Instead of relying too heavily on one tracer, we analyse multiple classes of Galactic tracers whose samples are dominated by objects discovered in recent decades mostly via infrared and radio surveys. Here we discuss the different classes of Galactic tracers.

Infrared Dark Clouds, Infrared Embedded Sources, Young Stellar Objects, Embedded Clusters, Open Clusters: Stars form in embedded clusters within molecular cloud complexes, which are typically within a few hundred pc of the Galactic plane. Embedded clusters disperse their dense gas on time-scale of $\lesssim 10^{6.5} \mathrm{yr}$, and the majority dissolve into unbound stellar associations on time-scale of $\sim 10^{7}$ yr (e.g. the OB associations), while $\sim 10 \%$ survive on time-scale of $\sim 10^{8-9}$ yr as open clusters Lada \& Lada 2003). All of these phenomena (molecular clouds, embedded clusters, young stellar objects, open clusters) generally trace the Galactic plane on larger scales, however local variations are obvious on smaller scales (e.g. the Gould Belt). 
A particular class of molecular cloud can be traced especially strongly along the Galactic plane. IRDCs were discovered in the mid 1990s with the Infrared Space Observatory (ISO) and Midcourse Space Experiment (MSX; Egan et al. 1998). They are observed in silhouette against the bright infrared emission of the Galactic plane and the most likely distance range to these clouds is $2-8 \mathrm{kpc}$ (Egan et al. 1998). These clouds are cold $(<25 \mathrm{~K})$ and known to be the sites of the earliest stages of star formation (Frieswijk \& Shipman 2010). We chose IRDCs as one of the tracers because of the abundance of IRDCs in the Galactic plane, particularly near the Galactic Centre (Chambers \& Yusef-Zadeh 2010).

HII Regions, HI Shells, Infrared Bubbles: Different flavours of "bubbles" pervade the interstellar medium in disc of the Galaxy, and can be found via observations at a wide range of wavelengths, especially the radio and infrared (e.g. Anderson et al. 2014 Simpson et al. 2012, Churchwell et al. 2006). H II regions are expanding high pressure regions of expanding ionized gas associated with luminous stars outputting copious amounts of UV radiation. Given their short dynamical time-scale and association with short-lived massive stars, they trace star formation and are concentrated in the spiral arms. Their strong radio emission enables the tracing of young massive stars at large distances (Paladini et al. 2003). Bubbles detected at infrared wavelengths mostly trace polycyclic aromatic hydrocarbon (PAH) emission, with cavities evacuated of dust. Only about a quarter are coincident with $\mathrm{H}$ II regions, and the majority appear to be dominated by mid to late B-type stars which do not excite H II regions (Churchwell et al. 2006).

AGB stars: Asymptotic giant branch (AGB) stars are luminous evolved stars undergoing extreme mass loss, and exhibiting very red infrared colors due to circumstellar dust. The AGB stars are categorized as "standard" O-rich and C-rich, and "extreme" (Robitaille et al. 2008). Such red giant stars trace the radial and vertical structure of the Galactic disc (Benjamin et al. 2005, Robitaille et al. 2008).

Supernovae Remnants: Supernova remnants (SNRs) are typically detected in optical, radio, and X-ray surveys as expanding regions of hot, shocked plasma associated with stellar explosions (Green 2009, 2014). They are morphologically classified as "shell" (S), "filled-centre" (F), or "composite" (C) structures in the radio. SNRs trace the Galactic plane fairly well in the 4th and 1st Galactic quadrants (see Fig. 3 of Green 2009), however there are only about 294 Galactic SNRs known (Green 2014), hence they provide a much smaller sample of tracers compared to our other categories of Galactic tracers.

\subsection{Catalogues}

We used three criteria to select our catalogues for analysis:

- They must be composed of the tracers mentioned in Section 2.1.
- The catalogues should map the Galactic plane reasonably evenly.

- For a given tracer, the corresponding catalogue should be the most recent and comprehensive one.

Coordinates for the tracers drawn from the catalogues are on the International Celestial Reference System (ICRS) for J2000. The selected catalogues are discussed in the following paragraphs.

Anderson et al. (2014) identified previously known Galactic H II regions and newly discovered candidate H II region using data from the all-sky WISE survey (Wright et al. 2010). This catalogue was created by visually inspecting WISE $12-\mu \mathrm{m}$ and $22-\mu \mathrm{m}$ images spanning the entire Galactic plane within the galactic latitude range $|b| \leq 8^{\circ}$. This catalogue consists of $8399 \mathrm{H}$ II regions (either previously known objects or new candidates).

Csengeri et al. (2014) identified embedded sources throughout the inner Galaxy using the ATLASGAL survey. The ATLASGAL survey imaged the Galactic Plane between Galactic longitude $-80^{\circ}<l<60^{\circ}$ and Galactic latitude $-2^{\circ}<b<1^{\circ}$ at $870-\mu \mathrm{m}$. These embedded sources are identified as the most prominent star-forming regions in the Galaxy. A total of 10861 compact sources were compiled in this catalogue.

Lumsden et al. (2013) assembled a large catalogue of statistically selected young massive protostars and $\mathrm{HII}$ regions. It was constructed using mid- and near-infrared data from the $M S X$ and $2 M A S S$ surveys, respectively (Egan et al. 1998 Skrutskie et al. 2006). The mid-IR survey was done in $b<5^{\circ}$ and $10^{\circ}<l<350^{\circ}$, and the catalogue contains 2811 objects.

Morales et al. (2013) catalogued 695 known open and embedded clusters in the inner Galaxy region. This catalogue was created from the ATLASGAL survey and the identified clusters that are within the range $|l| \leq 60^{\circ}$ and $|b| \leq 1.5^{\circ}$. Most of the open clusters lie within $\sim 1 \mathrm{kpc}$, and most of the embedded clusters lie within $\sim 2 \mathrm{kpc}$, hence the catalogue may carry some bias in that it is more heavily represented by local objects than the other catalogues.

Peretto \& Fuller (2009) identified and characterized a complete sample of Infrared Dark Clouds (IRDCs) in the GLIMPSE (Churchwell et al. 2009) and MIPSGAL (Carey et al. 2009) surveys undertaken by the Spitzer Space Telescope. Their survey analysed IRDCs in GLIMPSE 8- $\mu \mathrm{m}$ and MIPSGAL 24- $\mu \mathrm{m}$ images. The GLIMPSE survey ${ }^{2}$ is a conglomeration of multiple surveys that covered the entire $360^{\circ}$ longitude of the Galactic Plane and width ranging between $2^{\circ}-9^{\circ}$ in latitude. The MIPSGAL survey ${ }^{3}$ complemented the GLIMPSE legacy surveys and covered $|b|<1$ for $5<l<63$ and $298<l<355$ of the Galactic Plane and strips from $1<|b|<3$ for $-5<l<7$ at $24-\mu \mathrm{m}$ and $70-\mu \mathrm{m}$. The entries of the catalogue span $10^{\circ}<l<65^{\circ}$

\footnotetext{
2 See http://www.astro.wisc.edu/glimpse/all_GLIMPSE-dataAAS2013.pdf

s See http://mipsgal.ipac.caltech.edu/a_mipsgal.html
} 
and $|b|<1^{\circ}$. A total of 11303 clouds were analysed in this paper, of which $80 \%$ of the IRDCs were newly identified.

Robitaille et al. (2008) catalogued sources in the Galactic midplane from the GLIMPSE survey that have intrinsically red mid-infrared colours. These sources are dominated by high- and intermediate-mass young stellar objects (YSOs) and asymptotic giant branch (AGB) stars. Planetary nebulae and background galaxies together represent at most $2-3 \%$ of all the sources. This catalogue of 18,949 sources represents one of the largest compilations of YSOs and AGB stars tracing the Galactic plane.

Simpson et al. 2012) catalogued bubbles in the Spitzer GLIMPSE and MIPSGAL surveys. Their survey of the region $|l|<65^{\circ}$ and $|b|<1^{\circ}$ was based on visual inspection based on the online citizen scientists via "The Milky Way Project". This catalogue divides the bubbles into two categories: Large bubbles and Small bubbles. We calculated the position of the pole for the large and the small bubbles separately, and then for the combined samples. This catalogue consists of 5106 IR bubbles - 3744 large bubbles and 1362 small bubbles.

Green (2014) presented a catalogue of 294 Galactic supernovae remnants. This catalogue is heterogeneous and contains some objects which are probable SNR candidates. Most of the objects were detected via radio observation, and detection of distant SNRs is hampered by Galactic absorption. A few SNRs from this catalogue have high Galactic latitude value, however no attempt was made to clip the relatively small sample.

\section{ANALYSIS}

Three fundamental parameters define the GCS in spherical coordinates on the International Celestial Reference System (ICRS): the coordinate position of the NGP $(\alpha$ and $\delta$ ), and the position angle $\theta$ of the NGP with respect to the Galactic centre (defining the origin of the GCS), which defines longitude $\ell=0^{\circ}$. Following the principles of the 1958 IAU determination of the GCS, the problem can be reduced to one of solving for the best-fitting Galactic plane (the normal vector of which defines the NGP), and defining the origin of the GCS (determining the Galactic centre). We estimate these parameters using two different methods. The first "unconstrained" method calculates the parameters using least-squares fit for defining the Galactic plane through the samples of Galactic tracers discussed in Section 2 The second "constrained" method uses the methodology of the first, but constrains the NGP to be exactly $90^{\circ}$ away from Sgr A* by considering the position angle calculated in Section 3.1. Both of these methods are discussed in detail in the following subsections.

\subsection{Unconstrained NGP Solution}

In this method, we make the fundamental assumption that approximately half of the Galactic tracer are above and half

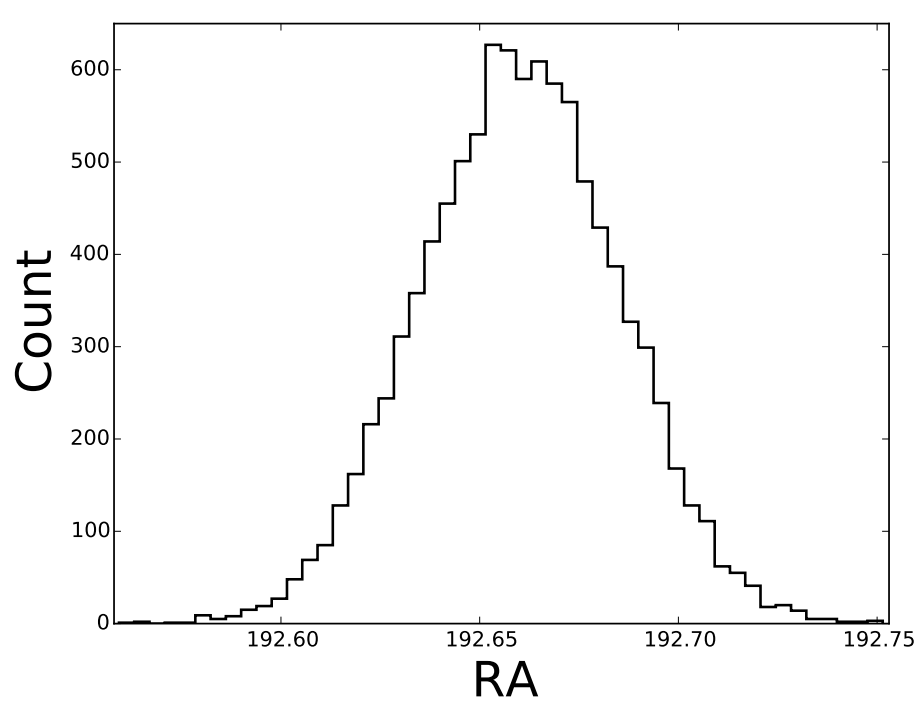

Figure 1. Histogram of $\alpha$ for the estimated North Galactic Pole using for the bootstrapped Robitaille et al. (2008) sample of candidate YSOs and AGB stars from the GLIMPSE survey.

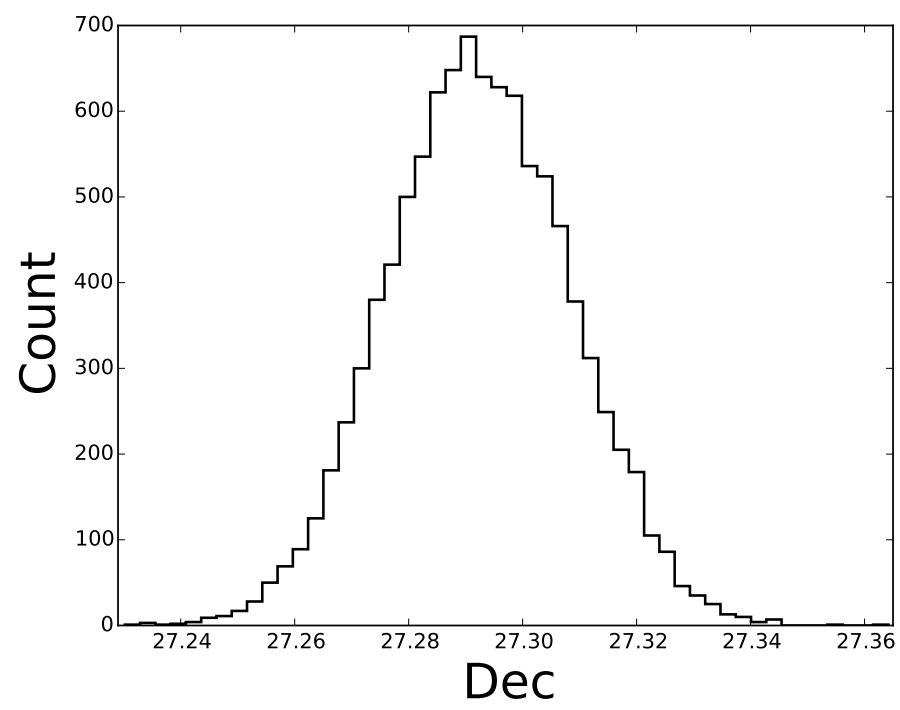

Figure 2. Histogram of $\delta$ for the estimated North Galactic Pole using for the bootstrapped Robitaille et al. (2008) sample of candidate YSOs and AGB stars from the GLIMPSE survey.

are below the Galactic plane. As discussed in Section 2 we have specifically selected catalogues for which this is a reasonable assumption. Thus if we do a least-squares fit analysis and calculate the plane of best-fitting of the tracers, this plane should coincide with the Galactic plane, and the normal vector to this plane should point towards the NGP. For this analysis, we convert the ICRS positions of the tracers into Cartesian coordinates on the equatorial system at unit distance. Thus, the equation of the plane is given by,

$z=A x+B y+C$

where $A, B, C$ are the normalized coefficients. 


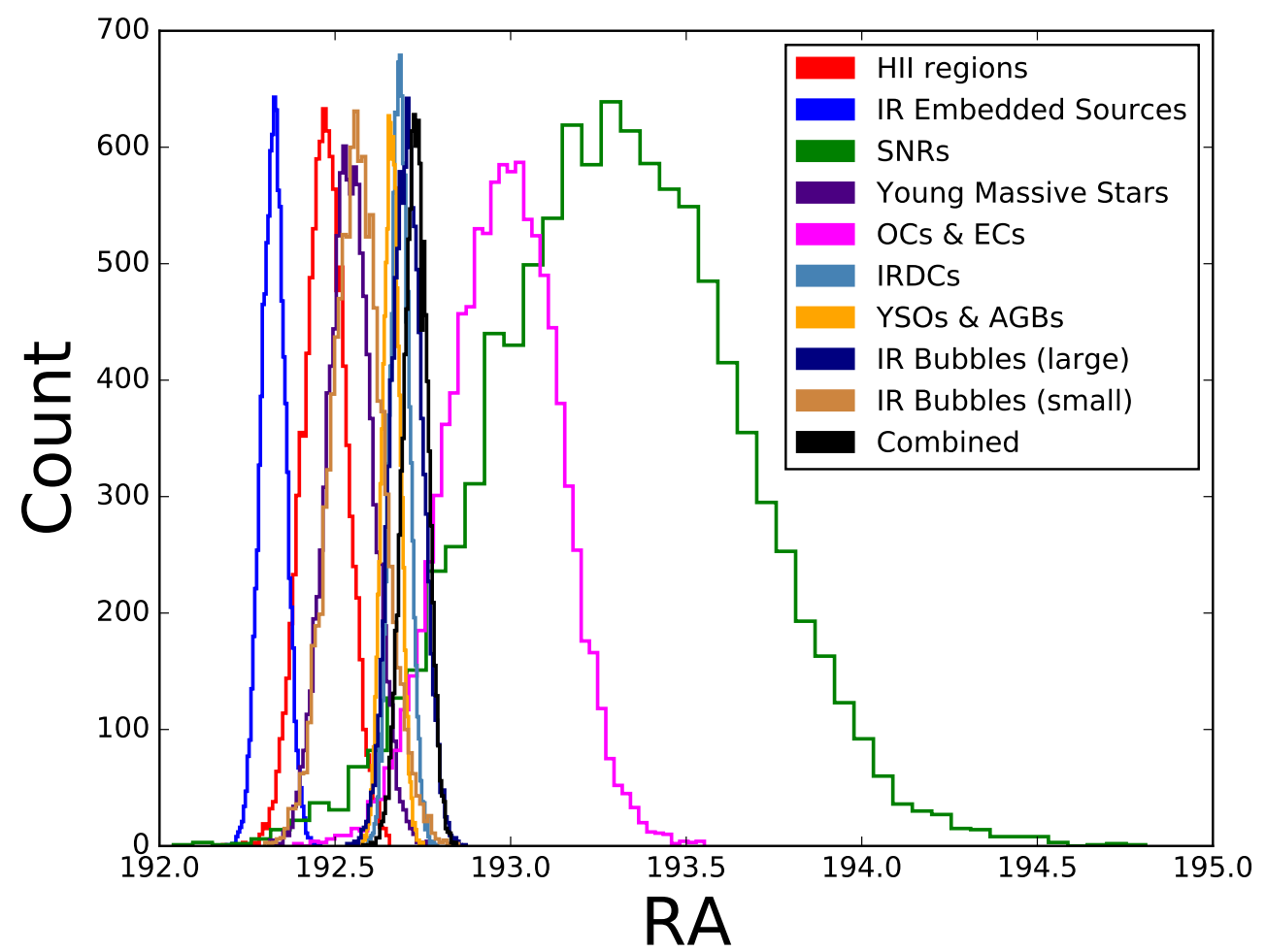

Figure 3. Histograms of $\alpha$ for the estimated North Galactic Pole for all of the samples analysed.

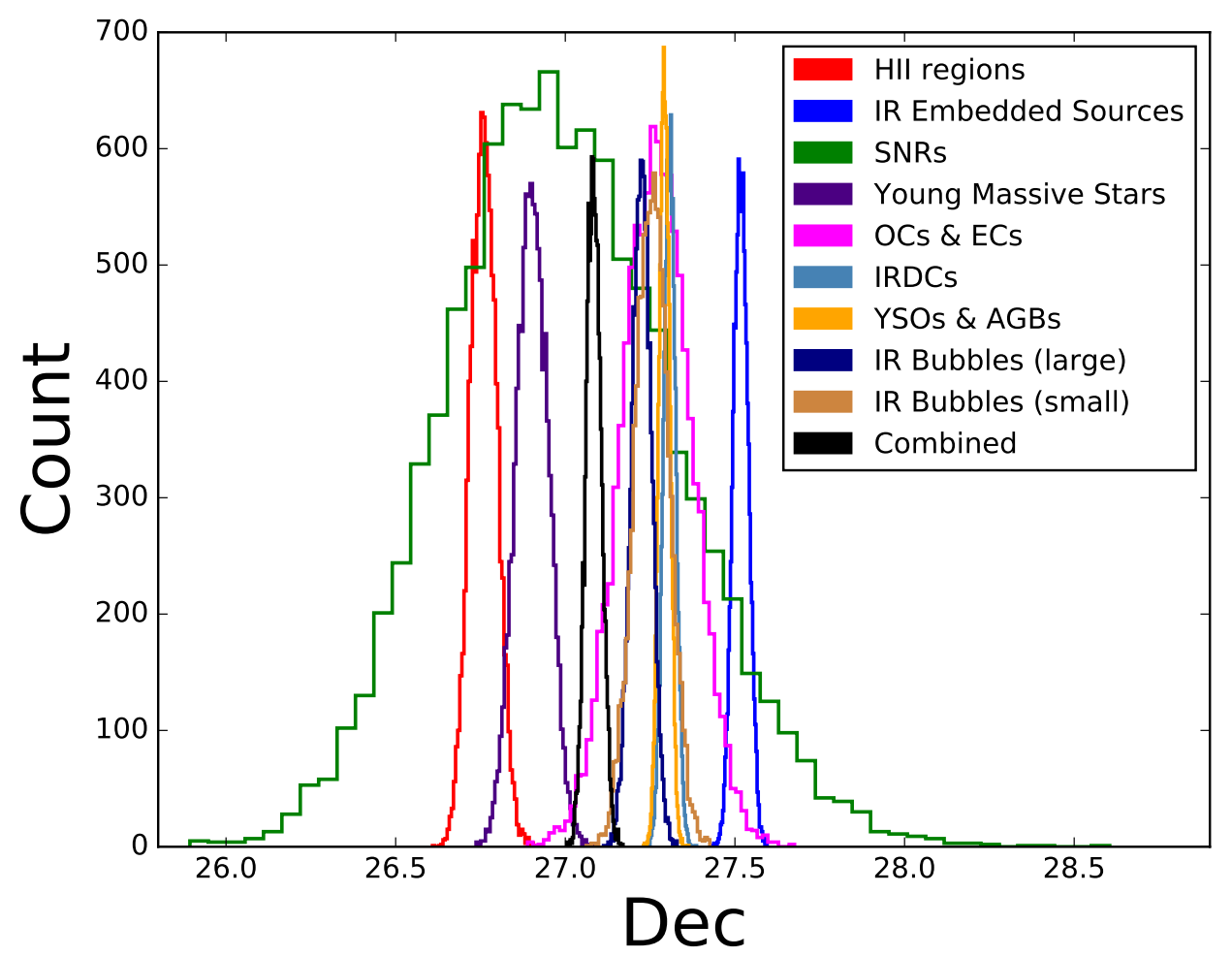

Figure 4. Histograms of $\delta$ for the estimated North Galactic Pole for all of the samples analysed. 
Table 1. Galactic Tracer Catalogues and Their Respective Estimated North Galactic Poles

\begin{tabular}{|c|c|c|c|c|c|}
\hline Catalogue & Tracer Type & $\mathrm{N}_{o b j}$ & $\begin{array}{c}\alpha \\
\left(^{\circ}\right)\end{array}$ & $\begin{array}{c}\delta \\
\left(^{\circ}\right)\end{array}$ & $\begin{array}{l}\text { PA } \\
\left(^{\circ}\right)\end{array}$ \\
\hline Anderson et al. 2014) & H II regions & 8399 & $192.471 \pm 0.061$ & $26.760 \pm 0.039$ & $122.812 \pm 0.028$ \\
\hline Peretto \& Fuller (2009) & IRDCs & 11303 & $192.684 \pm 0.028$ & $27.307 \pm 0.018$ & $122.908 \pm 0.013$ \\
\hline Robitaille et al. 2008) & YSO \& AGB stars & 18949 & $192.595 \pm 0.032$ & $27.358 \pm 0.014$ & $122.896 \pm 0.011$ \\
\hline Morales et al. 2013) & OCs \& ECs & 695 & $192.983 \pm 0.155$ & $27.266 \pm 0.104$ & $123.043 \pm 0.071$ \\
\hline Green 2014 & SNRs & 294 & $193.308 \pm 0.353$ & $26.992 \pm 0.335$ & $123.193 \pm 0.161$ \\
\hline Simpson et al. (2012) & IR Bubbles (Large) & 3744 & $192.705 \pm 0.043$ & $27.224 \pm 0.029$ & $122.917 \pm 0.020$ \\
\hline Simpson et al. (2012) & IR Bubbles (Small) & 1362 & $192.563 \pm 0.076$ & $27.255 \pm 0.052$ & $122.852 \pm 0.034$ \\
\hline \begin{tabular}{|l|l|l|} 
Simpson et al. 2012) \\
\end{tabular} & IR Bubbles (Combined) & 5106 & $192.673 \pm 0.038$ & $27.230 \pm 0.026$ & $122.903 \pm 0.018$ \\
\hline Csengeri et al. 2014) & IR Embedded Sources & 10861 & $192.325 \pm 0.034$ & $27.517 \pm 0.022$ & $122.742 \pm 0.016$ \\
\hline Lumsden et al. 2013 & Young Massive Stars & 2811 & $192.542 \pm 0.064$ & $26.903 \pm 0.049$ & $122.844 \pm 0.029$ \\
\hline Combined & & 58405 & $192.729 \pm 0.035$ & $27.084 \pm 0.023$ & $122.928 \pm 0.016$ \\
\hline
\end{tabular}

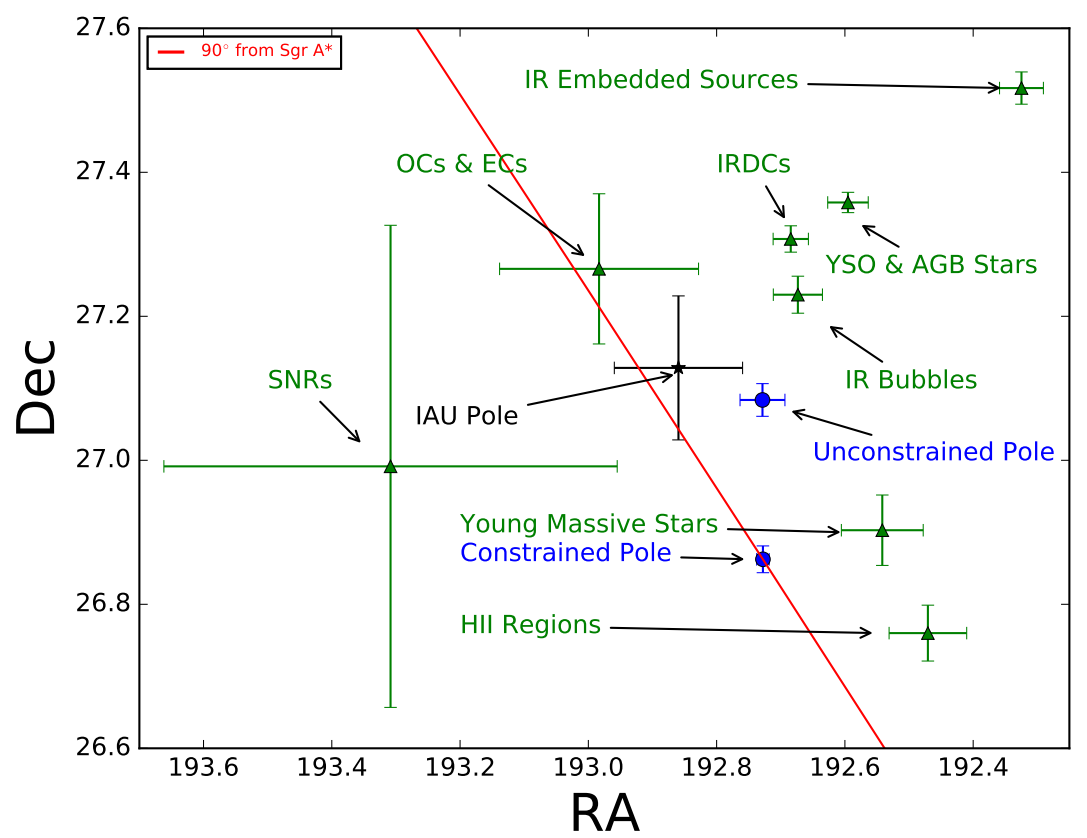

Figure 5. Estimation of the North Galactic Pole. This Dec vs. RA plot shows the locations and the $1 \sigma$ uncertainties of the NGPs for each of the catalogues (shown in green triangles), the proposed constrained and unconstrained NGPs (shown in blue circle) and the current IAU NGP (shown in black star). A line (shown in red) is also shown tracing exactly $90^{\circ}$ from Sgr A*, using its position from Reid \& Brunthaler (2004). Theoretically, the NGP should lie somewhere along this line.

The Cartesian coordinates of the tracers were then fitted to Equation 1 using the linalg.lstsq function from the Python package Scipy (Jones et al.2001-) to obtain the values of the normalized coefficients. From these coefficients, the coordinates of the NGP on the ICRS were calculated using the following relations,

$$
\begin{gathered}
\alpha_{N G P}=\tan ^{-1}(B / A) \\
\delta_{N G P}=\sin ^{-1}\left(A^{2}+B^{2}+1\right)^{-1 / 2}
\end{gathered}
$$

Because the tracers represent a tiny sample of the entire galactic population, we used the bootstrap resampling method (Efron 1979) to obtain a statistically robust estimate of the coefficients. Bootstrapping is the ideal resampling method to estimate the properties of the estimators because it is distribution-independent, provides a good estimation for small sample size, and is not affected by outliers (Adèr et al. 2008). By using the bootstrapping method, we generated $10^{4}$ virtual catalogues for each of the original catalogues. We fitted these virtual catalogues to 
Equations 12 and 3 to obtain $10^{4}$ values of $\left(\alpha_{N G P}, \delta_{N G P}\right)$.

Lastly, histograms of these values were generated and the histograms of the bootstrapped values appear to be Gaussian (e.g. see Figure 1 and Figure 2). The means and the variances of these histograms are quoted as the central values and uncertainties ${ }^{4}$ respectively in Table 1 Combined histograms of RA and Dec of the NGPs determined from all the catalogues are shown in Figure 3 and Figure 4 respectively. We used the ICRS coordinate of Sgr A* from Reid \& Brunthaler (2004) (listed in §1) to calculate the position angles.

The final "unconstrained' NGP and position angle were obtained by concatenating all the catalogues into one master catalogue and applying the same method as described above. These values are shown in bold in Table 1. The NGPs for the individual tracer samples (green triangles) and for all of the samples combined (blue circle) are plotted in Figure 5 along with the current (1958) IAU pole (black star). The red line plotted Figure 5 is the arc that lies exactly $90^{\circ}$ away from Sgr A*. We discuss the significance of our best-fitting unconstrained NGP lying more than $90^{\circ}$ away from Sgr A* in $\S 4.2$.

\subsection{Constrained NGP Solution}

In this method we constrained the pole to be exactly $90^{\circ}$ away from Sgr $\mathrm{A}^{*}$, along the great circle connecting Sgr $\mathrm{A}^{*}$ with the unconstrained NGP determined in $\S 3.1$. Consider a spherical triangle with the three vertices $\mathrm{A}, \mathrm{B}$ and $\mathrm{C}$ (as shown in Figure 6 5 where A corresponds to the North Celestial Pole (NCP), B corresponds to the NGP and C corresponds to Sgr $\mathrm{A}^{*}$. In this triangle the known values are:

$$
\begin{aligned}
& B C=a=90^{\circ} \\
& A C=b=90^{\circ}-\delta_{\mathrm{Sgr} A}=119^{\circ} .0078 \\
& \angle A B C=B=\text { position angle }=122^{\circ} .9280
\end{aligned}
$$

Since the two sides and the non-included angle is known, unique solutions exist if $a>\sin ^{-1}(\sin b \sin A)$, which is the case for our problem. Thus, we solved for $A$ and the side $c$ by using the spherical laws of sines and Napier's analogies:

$$
\begin{aligned}
& A=\sin ^{-1}\left(\frac{\sin a \sin B}{\sin b}\right)=73^{\circ} .6890 \\
& c=2 \tan ^{-1}\left[\tan \left(\frac{b-a}{2}\right) \frac{\sin \left(\frac{B+A}{2}\right)}{\sin \left(\frac{B-A}{2}\right)}\right]=63^{\circ} .1375
\end{aligned}
$$

From Figure 6, we used the following relations to solve for the NGP:

$\alpha_{\text {pole }}=\alpha_{\text {Sgr } A *}-A=192^{\circ} .7278$

$\delta_{\text {pole }}=90^{\circ}-c=26^{\circ} .8625$

After propagating the uncertainties, we obtained the following values for the "constrained" NGP:

4 All uncertainties for calculated quantities in this paper are $1 \sigma$.

5 Courtesy of http://star-www.st-and.ac.uk/

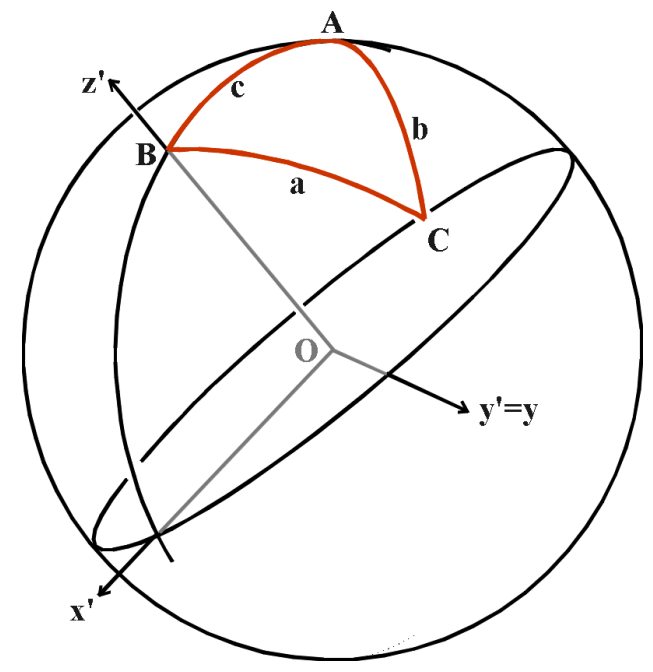

Figure 6. A spherical triangle denoting the locations of the NCP (point A), NGP (point B), Sagittarius A* (point C).

$$
\begin{aligned}
& \alpha_{N G P}^{\text {constrained }}=192^{\circ} .728 \pm 0^{\circ} .010 \\
& \delta_{N G P}^{\text {constrained }}=26^{\circ} .863 \pm 0^{\circ} .019
\end{aligned}
$$

\section{DISCUSSION}

\subsection{Comparison with the IAU Pole}

The IAU North Galactic Pole was determined nearly six decades ago, and heavily relied upon a single tracer (Galactic neutral hydrogen). In our analysis, we have estimated the NGP using eight different tracer samples, and the vast majority of the objects were unknown the astronomers decades ago. While most of the individual NGP determinations lie $>2 \sigma$ away from one another, our use of multiple tracer samples strengthens our case that our best-fitting constrained and unconstrained NGP estimates are not biased by any particular class of tracer (and is certainly not overly dependent on a single tracer like the IAU pole). Since the number of tracers used is a minuscule sample of the entire galactic tracer population, we used the bootstrapping re-sampling method to generate $10^{4}$ synthetic catalogues for each tracer type. Bootstrapping takes into account the potential distortion from poorly representative samples. Therefore, the number of tracers and the number of data points makes our analysis more statistically robust when compared to the current IAU definition of the NGP.

The current IAU pole is based on an estimate of the NGP with uncertainty $\sim 0{ }^{\circ} 1$ (Blaauw et al. 1960). In comparison, our proposed poles (both constrained and unconstrained) have an uncertainty of $\sim 0^{\circ} .03$ in $\mathrm{RA}$ and $\sim 0^{\circ} .02$ 
in Dec, so we have attained a factor of $\sim 4-5 \times$ improvement in accuracy for this important Galactic parameter.

\subsection{Comparison with recent papers}

In the recent years, three studies have addressed the issue of improving the Galactic coordinate system: Liu et al. (2011a), Liu et al. (2011b), and Ding et al. (2015). In this section, we compare our results with these papers in chronological order.

Liu et al. (2011a) noted that the GCS is rotating with respect to the ICRS, and this rotation factor cannot be removed easily due to the complexity of the fundamental catalogues, i.e. FK4 and FK5. As a temporary solution, they derived the rotation matrix from the equatorial to the GCS in the framework of the ICRS using the bias matrix. They also point out the necessity of placing Sgr $\mathrm{A}^{*}$ at the centre of the GCS and recommend that the IAU reconsiders the definition of the GCS, which may result in a more accurate depiction of the GCS. In their proposed method, they utilize the ICRS coordinates of Sgr A* and determine the coordinate of the NGP by setting the dot product of the ICRS coordinates of Sgr $\mathrm{A}^{*}$ and the ICRS coordinates of the NGP to be 0 , i.e. by setting the NGP exactly $90^{\circ}$ away from Sgr A* $^{*}$. For example, using Reid \& Brunthaler (2004)'s estimation of the RA and Dec of Sgr*, their estimation of the fundamental parameters are $-\alpha_{P}=12^{h} 51^{m} 36^{s} .7151981, \delta_{P}=27^{\circ} 06^{\prime} 11^{\prime} .193172, \theta=$ $123^{\circ} 0075021536$. While their paper brought up the issue of the limitation of the current GCS for the first time in decades, it only used one observational data point (Reid \& Brunthaler (2004)'s estimation of the coordinates of Sgr $\left.A^{*}\right)$ to estimate the coordinates of the NGP, without considering additional data (Liu et al. 2011b), thus lacking statistical rigour. Furthermore, in their following paper, Liu et al. (2011a) acknowledged that this estimation is off by several arcminutes from their estimation that used the bias matrix.

In contrast, we used nine different tracers that were bootstrapped $10^{4}$ times each, thus effectively our sample size consisted of 90,000 catalogues, making our estimates of the parameters and the uncertainties statistically more robust. We also used two methods to determine the pole, one which relies on the location of Sgr $\mathrm{A}^{*}$ and the other one does not. Thus, we show a more complete analysis of parameter estimation.

Liu et al. (2011b) later used the 2MASS near-IR Point Source Catalogue (Cutri et al. 2003, Skrutskie et al. 2006) and SPECFINDv2.0, a radio cross-identification catalogue, (Vollmer et al. 2010) in radio band to determine the fundamental parameters. They used two different methods to determine the fundamental parameters for each catalogue type - fixed z-axis, i.e. the z-axis of the GCS points in the direction of the NGP exactly, and fixed $x$-axis, i.e. the $\mathrm{x}$-axis of the GCS points in the direction of the Sgr $A^{*}$. They divided the catalogues in 360 longitudinal bins and for each of the fixed axis, they derived the parameters by determining the equatorial positions of the geometrical centres of each $1^{\circ}$-longitude bin. As a result, they published four possible sets of fundamental parameters. One of the catalogues, SPECFIND v2.0 is not evenly distributed and shows bias towards the northern hemisphere (Liu et al. 2011b). Liu et al. (2011b) acknowledged that the global structure of the SPECFIND catalogue had a strong effect on their results and they tried to remove biasing by restricting the data to $|b| \leq 5^{\circ}$. In addition, they ignored the weak interstellar extinction at the infrared and radio bands. Furthermore, as noted by Ding et al. (2015), Liu et al. (2011b) used only two catalogues, whereas in order to trace the full physical feature of the Milky Way, we need to consider more tracers. Ding et al. (2015) also noted that Liu et al. (2011b) did not offer any explicit recommendation for the GCS and that the methods used to find the galactic plane requires improvement.

Ding et al. (2015) conducted a similar analysis building on the methodology of Liu et al. (2011b). They used two all-sky surveys - the AKARI infrared all-sky survey ( $\mathrm{Mu}-$ rakami et al. 2007) and the WISE all-sky catalogue (Wright et al. (2010) - covering sources over six infrared bands between $3.4 \mu \mathrm{m}$ and $90 \mu \mathrm{m}$. Their methods are similar to that of Liu et al. (2011b) - fixed z-axis, i.e. the z-axis of the GCS points in the direction of the NGP exactly, and fixed $\mathrm{x}$-axis, i.e. the $\mathrm{x}$-axis of the GCS points in the direction of the $\operatorname{Sgr} \mathrm{A}^{*}$. To obtain the galactic plane, they created 360 bins, each corresponding to $1^{\circ}$ galactic longitude and calculated the medians of the bins, which was used to do the least-squares-fitting. The final results they proposed are: $\alpha_{P}=192^{\circ} .777, \delta_{P}=26^{\circ} .9298, \theta=122^{\circ} .95017$, based on the fixed $\mathrm{x}$-axis method. They argued that the $\mathrm{x}$-axis method is a better option because in this method they only calculated one parameter, the position angle, whereas in the fixed z-axis method, they calculated two parameters and so it maybe more prone to errors. Furthermore, when quoting the final values, they averaged the values obtained from the six wavelengths by applying equal weight to the values.

We have identified two limitations regarding the methodology of Ding et al. (2015). First, even though only one parameter, the position angle, was calculated for the fixed $\mathrm{x}$-axis method, Ding et al. (2015) acknowledged that their method was affected by singular points near the Galactic Centre and the anti-Galactic Centre because the value of the position angle became very unstable near these two longitudes, which resulted in them discarding many values (pages $7 \& 11$ of Ding et al. 2015). In comparison, we used bootstrapping resampling method to counter any possible outlier effect. As a result, we did not have to remove any data points from our analysis. We also note that since the measurements of the position angle at different wavelengths were affected differently and since there are different number of data points in different bands, applying equal weight to calculate the final value does not seem to be warranted. We further note that Ding et al. (2015) did not include any uncertainty in their measurement of the fundamental parameters. 
Table 2. Estimates of the Position Angle Between Sgr A* and NGP

\begin{tabular}{|c|c|c|}
\hline $\begin{array}{l}\text { Study } \\
\ldots\end{array}$ & $\begin{array}{l}\text { PA } \\
\text { (deg) }\end{array}$ & $\begin{array}{l}\text { unc. } \\
\text { (deg) }\end{array}$ \\
\hline IAU (calc. by Liu et al. 2011a) $^{1}$ & $122^{\circ} .93192526$ & $\ldots$ \\
\hline IAU (calc. by Liu et al. 2011a) ${ }^{1}$ & $122^{\circ} .93191857$ & $\ldots$ \\
\hline Liu et al. (2011a Sgr A* fixed) & $123^{\circ} .0075021536$ & $\ldots$ \\
\hline Ding et al. $(2015, z$-fixed $)$ & $122^{\circ} .86216$ & $\ldots$ \\
\hline Ding et al. $(2015, x$-fixed $)$ & $122^{\circ} .95017$ & $\ldots$ \\
\hline This Study & $122^{\circ} .928$ & $0^{\circ} .016$ \\
\hline
\end{tabular}

1 calculated by Liu et al. (2011a) using different transformations from FK4 (B1950.0) to FK5 (J2000.0) systems to the ICRS.

\subsection{Height of the Sun Above the Galactic Midplane}

Our unconstrained estimate of the NGP lies $90^{\circ} .120 \pm 0^{\circ} .029$, rather than $90^{\circ}$, away from the dynamical centre of the Galaxy ( $\left.\operatorname{Sgr} A^{*}\right)$. As Sgr A* lies a finite distance away $\left(R_{0}=8.2 \pm 0.1 \mathrm{kpc}\right.$; Bland-Hawthorn \& Gerhard 2016 this discrepancy can be explained by taking into account that the Sun has some finite distance above the Galactic midplane (i.e. the ratio of the Sun's height above the midplane compared to the distance to Sgr $A^{*}$ is very small, but not negligible or zero; see also $\S 3$ and Fig. 3 of Goodman et al. 2014). Hence there should be little surprise that the estimated NGP should be $>90^{\circ}$ from Sgr A*.

One can geometrically estimate the height of the Sun above the Galactic midplane using the estimate of the Galactocentric distance (to $\mathrm{Sgr} \mathrm{A}^{*}$ ) and the angular separation between the unconstrained estimate of the Galactic plane and Sgr A*. The geometry of this case is well illustrated in Fig. 3 of Goodman et al. (2014), and not reproduced here. The only difference with Fig. 3 of Goodman et al. (2014) is that we replace the "IAU mid-plane $b=0$ " with our unconstrained estimate of the Galactic plane using our combination of tracer samples $(\S 3.1)$. Combining the galactocentric distance from Bland-Hawthorn \& Gerhard (2016) with our estimate of the angular separation of our proposed NGP from Sgr A*, we estimate that the Sun would need to be $17.1 \pm 5.0 \mathrm{pc}$ above the Galactic midplane in order to explain Sgr A*'s angular distance below our best-fitting unconstrained Galactic Plane. This value is only $1.2 \sigma$ below the value adopted by Bland-Hawthorn \& Gerhard (2016) in their recent review on the Milky Way $(25 \pm 5 \mathrm{pc}$; based on the solar offset from the local disc midplane based on the SDSS photometric survey Jurić et al. 2008) In Table 3, we compile a list of 55 other published estimates of the Sun's height $z_{\odot}$ above the Galactic midplane, and plot the values in Figure 7. Combined with our new estimate, the ensemble of 56 estimates has a true median (Gott et al. 2001) of $z_{\odot} \simeq 17.4 \pm 1.9 \mathrm{pc}$, with a $95 \%$ confidence range of $15-22$

\footnotetext{
${ }^{6}$ Amusingly, the most recent best estimate of the Galactocentric distance $R_{0}$ advocated from the extensive literature survey of Bland-Hawthorn \& Gerhard (2016) of $8.2 \pm 0.1 \mathrm{kpc}$ is identical in value to that adopted in the works that helped define the 1958 IAU GCS (Blaauw et al. 1960).
}

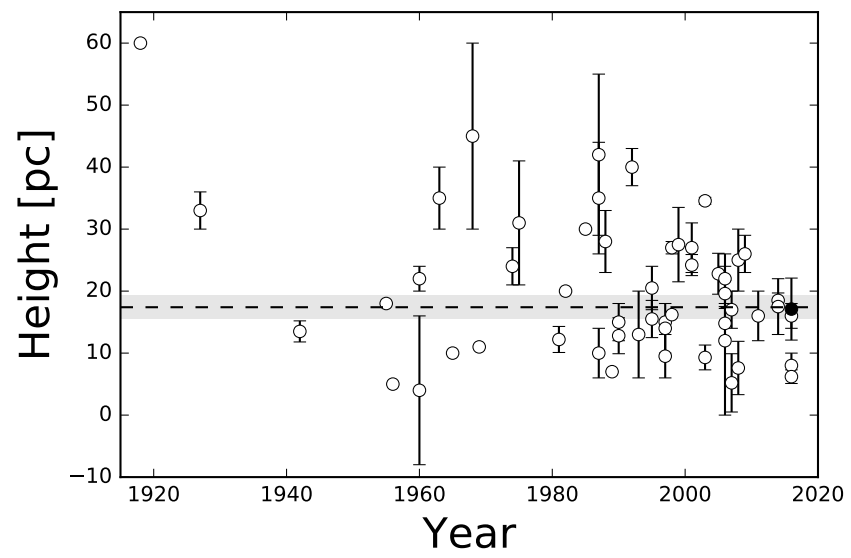

Figure 7. Plot of publication year vs. estimated height of the Sun above the Galactic plane $z_{\odot}$ (in parsecs) calculated by various studies since 1918, except Kreiken (1926)'s estimate of 250 pc. The filled dot represents our estimate, the open dots represent values determined by different studies, the dashed line represents the median (17.4 pc) and the grey-shaded area the uncertainty range $( \pm 1.9 \mathrm{pc})$ of all the measurements. The published estimates are listed in Table 3

pc, suggesting that our estimate is very close to the locus of previous estimates. Given the Sun's vertical velocity with respect to the Local Standard of Rest $\left(7.25 \pm 0.36 \mathrm{~km} \mathrm{~s}^{-1}\right.$; Schönrich et al. 2010), this suggests that the Sun passed through the Galactic midplane approximately $2.5 \pm 0.3 \mathrm{Myr}$ ago, coincidently at the beginning of the Pleistocene epoch (2.58 Myr ago; Cohen et al. 2015).

\section{CONCLUSIONS}

Given the importance of the Galactic Coordinate System in the study of galactic structure and kinematics, a more accurate representation of the GCS based on the physical markers of the Galaxy may be overdue. We have derived the fundamental parameters that define the Galactic Coordinate System, i.e. the RA and Dec of the NGP and the position angle of the NGP with respect to the Galactic Centre at the North Celestial Pole, using two different analyses on large samples of Galactic tracers. In the unconstrained method, we used eight galactic tracers to determine the plane of bestfitting to determine the location of the NGP, without taking the location of Sgr $\mathrm{A}^{*}$ into account. The parameters determined using this method are: $\alpha_{N G P}=192^{\circ} .729 \pm 0^{\circ} .035$, $\delta_{N G P}=27^{\circ} .084 \pm 0^{\circ} .023$ and $\theta=122^{\circ} .928 \pm 0^{\circ} .016$.

Using this first method, the NGP lies $90^{\circ} .120 \pm 0^{\circ} .029$ away from Sgr $\mathrm{A}^{*}$, instead of being exactly $90^{\circ}$ away. We explain this discrepancy as a result of the height of the Sun above the Galactic midplane. Using this discrepancy, we have independently estimated the the height of the Sun above the Galactic midplane to be $z_{\odot}=17.1 \pm 5.0$. Our new estimate of the height of the Sun above the Galactic midplane is similar to the true median estimate based on 56 published estimates $(17.4 \pm 1.9 \mathrm{pc})$.

Using the "constrained" methodology, we solved for an estimate of the NGP which would lie exactly $90^{\circ}$ away from Sgr A*, along the great circle connecting $\mathrm{Sgr} \mathrm{A}^{*}$ with 
Table 3. A Century of Estimates of the Sun's Height Above the Galactic Mid-Plane

\begin{tabular}{|c|c|c|}
\hline Reference & Description & $z_{\odot}(\mathrm{pc})$ \\
\hline Shapley 1918 & Globular clusters & 60 \\
\hline Kreiken $(\overline{1926})$ & Local stellar system & 250 \\
\hline Gerasimovic \& Luyten (1927) & Cepheids, O-, B-, c- \& ac-type stars & $33 \pm 3$ \\
\hline van Tulder $(1942)$ & Cepheids, planetary neb., c-, O-, B-, and WR-type stars & $13.5 \pm 1.7$ \\
\hline van Rhijn (1955) & A-type stars & 18 \\
\hline van Rhijn $(1956)$ & K-type stars & 5 \\
\hline Gum et al. $(1960)$ & H I gas & $4 \pm 12$ \\
\hline Blaauw $(1960)$ & OB-type stars \& Cepheids & $22 \pm 2$ \\
\hline Kraft \& Schmidt (1963) & Cepheids & $35 \pm 5(30-40)$ \\
\hline Elvius (1965) & AFGK-type stars & 10 \\
\hline Fernie $(\overline{1968)}$ & Cepheids & $45 \pm 15$ \\
\hline de Vaucouleurs \& Malik (1969) & Galactic absorbing layer & 11 \\
\hline Stothers \& Frogel (1974) & OB-type stars & $24 \pm 3$ \\
\hline Stenholm $(1975)$ & WR stars & $31 \pm 10$ \\
\hline Toller (1981) & Pioneer 10 observation of background starlight & $12.2 \pm 2.1$ \\
\hline Lynga (1982) & Open clusters & 20 \\
\hline Magnani et al. (1985) & Molecular gas & 30 \\
\hline Stobie \& Ishida (1987) & $U B V$ star counts & $42 \pm 13$ \\
\hline Caldwell \& Coulson (1987) & Cepheids & $35 \pm 9^{a}$ \\
\hline Pandey \& Mahra (1987) & Interstellar matter & $10 \pm 4$ \\
\hline Pandey et al. (1988) & Open clusters & $28 \pm 5$ \\
\hline Ratnatunga et al. (1989) & Stars in Yale Bright Star Catalogue & 7 \\
\hline Conti \& Vacca $(1990)$ & Wolf-Rayet stars & $15 \pm 3$ \\
\hline Toller $(1990)$ & Background light \& interstellar dust & $12.8 \pm 2.9$ \\
\hline Yamagata \& Yoshii $(1992)$ & $U B V$ star-count & $40 \pm 3$ \\
\hline Brand \& Blitz $(1993)$ & Local molecular clouds & $13 \pm 7$ \\
\hline Hammersley et al. (1995) & Two Micron Galactic Survey, IRAS and COBE & $15.5 \pm 3$ \\
\hline Cohen $(1995)$ & IRAS point source \& FAUST catalogues & $15.5 \pm 0.5$ \\
\hline Humphreys \& Larsen (1995) & Optical star counts & $20.5 \pm 3.5$ \\
\hline $\mathrm{Ng}$ et al. $(1997)$ & Star counts \& colors & 15 \\
\hline Binney et al. (1997) & COBE all-sky survey & $14 \pm 4$ \\
\hline Reed (1997) & OB-type stars & $9.5 \pm 3.5(6-13)$ \\
\hline Freudenreich (1998) & Diffuse infrared background & $16.2^{a}$ \\
\hline Mendez \& van Altena (1998) & Interstellar dust & $27 \pm 1$ \\
\hline Chen et al. (1999) & COBE \& IRAS all-sky reddening map & $27.5 \pm 6.0$ \\
\hline Maíz-Apellániz (2001) & OB-type stars & $24.2 \pm 1.7$ \\
\hline Chen et al. $(2001)$ & SDSS star counts & $27 \pm 4$ \\
\hline Branham (2003) & Hipparcos stars except OB stars & $34.56 \pm 0.56$ \\
\hline Paladini et al. (2003) & H iI regions & $9.3 \pm 2(7.3-11.3)$ \\
\hline Joshi (2005) & Reddening of open clusters & $22.8 \pm 3.3$ \\
\hline Reed $(2006)$ & OB-type stars & $19.6 \pm 2.1$ \\
\hline Piskunov et al. (2006) & Open clusters & $22 \pm 4$ \\
\hline Elias et al. $(2006)$ & OB-type stars & $12 \pm 12$ \\
\hline Bonatto et al. (2006) & Open clusters & $14.8 \pm 2.4$ \\
\hline van Leeuwen (2007) & Hipparcos A-/F-type stars & $5.2 \pm 4.7$ \\
\hline Joshi $(2007)$ & Young open clusters \& OB-type stars & $17 \pm 3$ \\
\hline Kong \& Zhu (2008) & OB-type \& horizontal branch stars & $7.6 \pm 4.3^{b}$ \\
\hline Jurić et al. $(2008)$ & SDSS stellar density distribution & $25 \pm 5$ \\
\hline Majaess et al. (2009) & Cepheids & $26 \pm 3$ \\
\hline Liu \& Zhu (2011) & Open clusters & $16 \pm 4$ \\
\hline Buckner \& Froebrich (2014) & Open clusters & $18.5 \pm 1.2$ \\
\hline Olausen \& Kaspi (2014) & Magnetar and magnetar candidates & $17.5 \pm 4.5(13-22)$ \\
\hline Bobylev \& Bajkova 2016a) & OB-, Wolf-Rayet-type stars, Cepheids \& H II regions & $16 \pm 2$ \\
\hline Bobylev \& Bajkova $(\overline{2016 b})$ & H II regions, masers \& molecular clouds & $8 \pm 2(6-10)$ \\
\hline Joshi et al. $(2016)$ & Open clusters & $6.2 \pm 1.1$ \\
\hline This study & Sgr A* offset from Galactic plane & $\mathbf{1 7 . 1} \pm \mathbf{5 . 0}$ \\
\hline True Median $z_{\odot}$ & & $17.4 \pm 1.9$ \\
\hline
\end{tabular}

Notes:

${ }^{a}$ : Estimated value for $R_{0}=8.2 \mathrm{kpc}$.

${ }^{b}$ : Weighted mean using their two estimates $(3.5 \pm 5.4 \mathrm{pc}, 15.2 \pm 7.3 \mathrm{pc})$. 
the unconstrained NGP. The parameters determined using this method are: $\alpha_{N G P}=192^{\circ} .7278 \pm 0^{\circ} .0098, \delta_{N G P}=$ $26^{\circ} .863 \pm 0^{\circ} .019$ and $\theta=122^{\circ} .928 \pm 0^{\circ} .016$. In both the methods, the uncertainties in the position of the NGP are $\sim 4-5 \times$ smaller than those of the original IAU estimate from the 1950 s.

Which solution is the "best" may depend on the application. For a simple 2D Galactic coordinate system, the constrained (2nd) solution which forces the poles to be exactly $90^{\circ}$ from $\mathrm{Sgr} \mathrm{A}^{*}$ may be a more reasonable option as it anchors the origin to the dynamical centre of the Galaxy. However, for Galactic kinematic calculations, one would want to solve for the motion of the stars (or Sun) in a Galactocentric cylindrical coordinate system, preferably one which takes into account the Sun's height above the Galactic midplane $\left(z_{\odot}=17 \mathrm{pc}\right)$. In this case, the parameters for the unconstrained NGP should be more the more relevant solution. As the Sun lies a finite distance above the Galactic midplane, one should not expect the dynamical centre of the Galaxy (Sgr $A^{*}$ ) to appear precisely $90^{\circ}$ from the normal to the best-fitting Galactic plane determined using the positions of Galactic tracers as seen from the solar system. In this case, one would expect Galactic tracers to define a Galactic plane projected asymptotically to lie some small angle above Sgr A*, as is seen. With the recent release of the first data release of Gaia astrometry, it will be interesting to compare geometric estimates of the NGP based on Galactic tracers with dynamical estimates based on stellar kinematics.

\section{ACKNOWLEDGEMENTS}

MTK participated in the summer 2014 University of Rochester REU program supported by PHY-1156339. MTK and EEM acknowledges support from NSF awards AST1313029. EEM acknowledges support from the NASA NExSS program. We thank Eric Feigelson, Segev BenZvi, Sanha Cheong, and Alec Kirkley for discussions on statistics, and Matthias Steinmetz for discussions at the 2015 IAU meeting on the Milky Way.

\section{REFERENCES}

Adèr H., Mellenbergh G., Hand D., 2008, Advising on research methods: a consultant's companion. Johannes van Kessel Publishing

Anderson L. D., Bania T. M., Balser D. S., Cunningham V., Wenger T. V., Johnstone B. M., Armentrout W. P., 2014, ApJS, 212, 1

Benjamin R. A., Churchwell E., Babler B. L., Indebetouw R., Meade M. R., Whitney B. A., Watson C., Wolfire M. G., Wolff M. J., Ignace R., Bania T. M., Bracker S., Clemens D. P., Chomiuk L., Cohen M., Dickey J. M., Jackson J. M., Kobulnicky H. A., Mercer E. P., Mathis J. S., Stolovy S. R., Uzpen B., 2005, ApJ, 630, L149

Binney J., Gerhard O., Spergel D., 1997, MNRAS, 288, 365 Blaauw A., 1960, MNRAS, 121, 164

Blaauw A., Gum C. S., Pawsey J. L., Westerhout G., 1960, MNRAS, 121, 123

Bland-Hawthorn J., Gerhard O., 2016, ArXiv e-prints
Bobylev V. V., Bajkova A. T., 2016a, Astronomy Letters, 42,1

Bobylev V. V., Bajkova A. T., 2016b, Astronomy Letters, 42, 182

Bonatto C., Kerber L. O., Bica E., Santiago B. X., 2006 , A\&A, 446, 121

Brand J., Blitz L., 1993, A\&A, 275, 67

Branham Jr. R. L., 2003, Ap\&SS, 288, 417

Brown R. L., 1982, ApJ, 262, 110

Buckner A. S. M., Froebrich D., 2014, MNRAS, 444, 290

Caldwell J. A. R., Coulson I. M., 1987, AJ, 93, 1090

Carey S. J., Noriega-Crespo A., Mizuno D. R., Shenoy S., Paladini R., Kraemer K. E., Price S. D., Flagey N., Ryan E., Ingalls J. G., Kuchar T. A., Pinheiro Gonçalves D., Indebetouw R., Billot N., Marleau F. R., Padgett D. L., Rebull L. M., Bressert E., Ali B., Molinari S., Martin P. G., Berriman G. B., Boulanger F., Latter W. B., MivilleDeschenes M. A., Shipman R., Testi L., 2009, PASP, 121, 76

Chambers E., Yusef-Zadeh F., 2010, Characterizing Star Formation in Galactic Center IRDCs. ATNF Proposal

Chen B., Figueras F., Torra J., Jordi C., Luri X., Galadí-

Enríquez D., 1999, A\&A, 352, 459

Chen B., Stoughton C., Smith J. A., Uomoto A., Pier J. R., Yanny B., Ivezić Ž., York D. G., Anderson J. E., Annis J., Brinkmann J., Csabai I., Fukugita M., Hindsley R., Lupton R., Munn J. A., SDSS Collaboration, 2001, ApJ, 553,184

Churchwell E., Babler B. L., Meade M. R., Whitney B. A., Benjamin R., Indebetouw R., Cyganowski C., Robitaille T. P., Povich M., Watson C., Bracker S., 2009, PASP, 121, 213

Churchwell E., Povich M. S., Allen D., Taylor M. G., Meade M. R., Babler B. L., Indebetouw R., Watson C., Whitney B. A., Wolfire M. G., Bania T. M., Benjamin R. A., Clemens D. P., Cohen M., Cyganowski C. J., Jackson J. M., Kobulnicky H. A., Mathis J. S., Mercer E. P., Stolovy S. R., Uzpen B., Watson D. F., Wolff M. J., 2006, ApJ, 649, 759

Cohen K. M., Finney S. C., Gibbard P. L., 2015, International chronostratigraphic chart. http://www.stratigraphy.org/ICSchart/ ChronostratChart2015-01.pdf international Commission on Stratigraphy

Cohen M., 1995, ApJ, 444, 874

Conti P. S., Vacca W. D., 1990, AJ, 100, 431

Csengeri T., Urquhart J. S., Schuller F., Motte F., Bontemps S., Wyrowski F., Menten K. M., Bronfman L., Beuther H., Henning T., Testi L., Zavagno A., Walmsley M., 2014, A\&A, 565, A75

Cutri R. M., Skrutskie M. F., van Dyk S., et al., 2003, 2MASS Point Source Catalogue (available at http://www.ipac.caltech.edu/2mass/)

de Vaucouleurs G., Malik G. M., 1969, MNRAS, 142, 387

Ding P.-J., Zhu Z., Liu J.-C., 2015, Research in Astronomy and Astrophysics, 15, 1045

Efron B., 1979, The Annals of Statistics, 7, 1

Egan M. P., Shipman R. F., Price S. D., Carey S. J., Clark F. O., Cohen M., 1998, ApJ, 494, L199

Elias F., Cabrera-Caño J., Alfaro E. J., 2006, AJ, 131, 2700

Elvius T., 1965, in Galactic Structure, Blaauw A., Schmidt M., eds., p. 41 
Fernie J. D., 1968, AJ, 73, 995

Freudenreich H. T., 1998, ApJ, 492, 495

Frieswijk W. W. F., Shipman R. F., 2010, A\&A, 515, A51

Gerasimovic B. P., Luyten W. J., 1927, Proceedings of the

National Academy of Science, 13, 387

Goodman A. A., Alves J., Beaumont C. N., Benjamin R. A., Borkin M. A., Burkert A., Dame T. M., Jackson J., Kauffmann J., Robitaille T., Smith R. J., 2014, ApJ, 797, 53

Gott J. R. I., Vogeley M. S., Podariu S., Ratra B., 2001, ApJ, 549, 1

Green D. A., 2009, Bulletin of the Astronomical Society of India, 37, 45

Green D. A., 2014, Bulletin of the Astronomical Society of India, 42, 47

Gum C. S., Kerr F. J., Westerhout G., 1960, MNRAS, 121, 132

Gum C. S., Pawsey J. L., 1960, MNRAS, 121, 150

Hammersley P. L., Garzon F., Mahoney T., Calbet X., 1995, MNRAS, 273, 206

Herschel W., 1785, Philosophical Transactions of the Royal Society of London Series I, 75, 213

Humphreys R. M., Larsen J. A., 1995, AJ, 110, 2183

Jones E., Oliphant T., Peterson P., et al., 2001-, SciPy: Open source scientific tools for Python. [Online; accessed 2016-05-22]

Joshi Y. C., 2005, MNRAS, 362, 1259

Joshi Y. C., 2007, MNRAS, 378, 768

Joshi Y. C., Dambis A., Pandey A. K., Joshi S., 2016, ArXiv e-prints

Jurić M., Ivezić Ž., Brooks A., Lupton R. H., Schlegel D., Finkbeiner D., Padmanabhan N., Bond N., Sesar B., Rockosi C. M., Knapp G. R., Gunn J. E., Sumi T., Schneider D. P., Barentine J. C., Brewington H. J., Brinkmann J., Fukugita M., Harvanek M., Kleinman S. J., Krzesinski J., Long D., Neilsen Jr. E. H., Nitta A., Snedden S. A., York D. G., 2008, ApJ, 673, 864

Kong D. L., Zhu Z., 2008, Acta Astronomica Sinica, 49, 224

Kraft R. P., Schmidt M., 1963, ApJ, 137, 249

Kreiken E. A., 1926, MNRAS, 86, 665

Lada C. J., Lada E. A., 2003, ARA\&A, 41, 57

Lindegren L., Lammers U., Bastian U., Hernández J., Klioner S., Hobbs D., Bombrun A., Michalik D., RamosLerate M., Butkevich A., Comoretto G., Joliet E., Holl B., Hutton A., Parsons P., Steidelmüller H., Abbas U., Altmann M., Andrei A., Anton S., Bach N., Barache C., Becciani U., Berthier J., Bianchi L., Biermann M., Bouquillon S., Bourda G., Brüsemeister T., Bucciarelli B., Busonero D., Carlucci T., Castañeda J., Charlot P., Clotet M., Crosta M., Davidson M., de Felice F., Drimmel R., Fabricius C., Fienga A., Figueras F., Fraile E., Gai M., Garralda N., Geyer R., González-Vidal J. J., Guerra R., Hambly N. C., Hauser M., Jordan S., Lattanzi M. G., Lenhardt H., Liao S., Löffler W., McMillan P. J., Mignard F., Mora A., Morbidelli R., Portell J., Riva A., Sarasso M., Serraller I., Siddiqui H., Smart R., Spagna A., Stampa U., Steele I., Taris F., Torra J., van Reeven W., Vecchiato A., Zschocke S., de Bruijne J., Gracia G., Raison F., Lister T., Marchant J., Messineo R., Soffel M., Osorio J., de Torres A., O'Mullane W., 2016, ArXiv e-prints

Liu J., Zhu Z., 2011, in Astronomical Society of the Pacific
Conference Series, Vol. 451, 9th Pacific Rim Conference on Stellar Astrophysics, Qain S., Leung K., Zhu L., Kwok S., eds., p. 339

Liu J.-C., Zhu Z., Zhang H., 2011a, A\&A, 526, A16

Liu J.-C., Zhu Z., Hu B., 2011b, A\&A, 536, A102

Lumsden S. L., Hoare M. G., Urquhart J. S., Oudmaijer R. D., Davies B., Mottram J. C., Cooper H. D. B., Moore T. J. T., 2013, ApJS, 208, 11

Lynga G., 1982, A\&A, 109, 213

Magnani L., Blitz L., Mundy L., 1985, ApJ, 295, 402

Maíz-Apellániz J., 2001, AJ, 121, 2737

Majaess D. J., Turner D. G., Lane D. J., 2009, MNRAS, 398, 263

Mendez R. A., van Altena W. F., 1998, A\&A, 330, 910

Morales E. F. E., Wyrowski F., Schuller F., Menten K. M., 2013, A\&A, 560, A76

Murakami H., Baba H., Barthel P., Clements D. L., Cohen M., Doi Y., Enya K., Figueredo E., Fujishiro N., Fujiwara H., Fujiwara M., Garcia-Lario P., Goto T., Hasegawa S., Hibi Y., Hirao T., Hiromoto N., Hong S. S., Imai K., Ishigaki M., Ishiguro M., Ishihara D., Ita Y., Jeong W.-S., Jeong K. S., Kaneda H., Kataza H., Kawada M., Kawai T., Kawamura A., Kessler M. F., Kester D., Kii T., Kim D. C., Kim W., Kobayashi H., Koo B. C., Kwon S. M., Lee H. M., Lorente R., Makiuti S., Matsuhara H., Matsumoto T., Matsuo H., Matsuura S., Müller T. G., Murakami N., Nagata H., Nakagawa T., Naoi T., Narita M., Noda M., Oh S. H., Ohnishi A., Ohyama Y., Okada Y., Okuda H., Oliver S., Onaka T., Ootsubo T., Oyabu S., Pak S., Park Y.-S., Pearson C. P., Rowan-Robinson M., Saito T., Sakon I., Salama A., Sato S., Savage R. S., Serjeant S., Shibai H., Shirahata M., Sohn J., Suzuki T., Takagi T., Takahashi H., Tanabé T., Takeuchi T. T., Takita S., Thomson M., Uemizu K., Ueno M., Usui F., Verdugo E., Wada T., Wang L., Watabe T., Watarai H., White G. J., Yamamura I., Yamauchi C., Yasuda A., 2007, PASJ, 59, S369

Ng Y. K., Bertelli G., Chiosi C., Bressan A., 1997, A\&A, 324,65

Ohlsson J., 1932, Annals of the Observatory of Lund, 3, 1

Ohlsson J., Reiz A., Torgård I., 1956, Lund Observatory

Tables for the Conversion of Galactic Into Equatorial Coordinates for the Epoch 1958.0

Olausen S. A., Kaspi V. M., 2014, ApJS, 212, 6

Oort J. H., Rougoor G. W., 1960, MNRAS, 121, 171

Paladini R., Burigana C., Davies R. D., Maino D., Bersanelli M., Cappellini B., Platania P., Smoot G., 2003, A\&A, 397, 213

Pandey A. K., Bhatt B. C., Mahra H. S., 1988, A\&A, 189, 66

Pandey A. K., Mahra H. S., 1987, MNRAS, 226, 635

Peretto N., Fuller G. A., 2009, A\&A, 505, 405

Piskunov A. E., Kharchenko N. V., Röser S., Schilbach E., Scholz R.-D., 2006, A\&A, 445, 545

Ratnatunga K. U., Bahcall J. N., Casertano S., 1989, ApJ, 339, 106

Reed B. C., 1997, PASP, 109, 1145

Reed B. C., 2006, JRASC, 100, 146

Reid M. J., Brunthaler A., 2004, ApJ, 616, 872

Robitaille T. P., Meade M. R., Babler B. L., Whitney B. A., Johnston K. G., Indebetouw R., Cohen M., Povich M. S., Sewilo M., Benjamin R. A., Churchwell E., 2008, AJ, 136, 2413 
Schönrich R., Binney J., Dehnen W., 2010, MNRAS, 403, 1829

Shapley H., 1918, PASP, 30, 42

Simpson R. J., Povich M. S., Kendrew S., Lintott C. J., Bressert E., Arvidsson K., Cyganowski C., Maddison S., Schawinski K., Sherman R., Smith A. M., Wolf-Chase G., 2012, MNRAS, 424, 2442

Skrutskie M. F., Cutri R. M., Stiening R., Weinberg M. D., Schneider S., Carpenter J. M., Beichman C., Capps R., Chester T., Elias J., Huchra J., Liebert J., Lonsdale C., Monet D. G., Price S., Seitzer P., Jarrett T., Kirkpatrick J. D., Gizis J. E., Howard E., Evans T., Fowler J., Fullmer L., Hurt R., Light R., Kopan E. L., Marsh K. A., McCallon H. L., Tam R., Van Dyk S., Wheelock S., 2006, AJ, 131,1163

Stenholm B., 1975, A\&A, 39, 307

Stobie R. S., Ishida K., 1987, AJ, 93, 624

Stothers R., Frogel J. A., 1974, AJ, 79, 456

Toller G. N., 1981, PhD thesis, State University of New York, Stony Brook.

-, 1990, in IAU Symposium, Vol. 139, The Galactic and Extragalactic Background Radiation, Bowyer S., Leinert C., eds., pp. 21-34

van Leeuwen F., ed., 2007, Astrophysics and Space Science Library, Vol. 350, Hipparcos, the New Reduction of the Raw Data

van Rhijn E. A., 1955, Pub. Kapteyn Astr. Lab Groningen, 57

van Rhijn E. A., 1956, Pub. Kapteyn Astr. Lab Groningen, 59

van Tulder J. J. M., 1942, Bull. Astron. Inst. Netherlands, 9, 315

Vollmer B., Gassmann B., Derrière S., Boch T., Louys M., Bonnarel F., Dubois P., Genova F., Ochsenbein F., 2010, A\&A, 511, A53

Wright E. L., Eisenhardt P. R. M., Mainzer A. K., Ressler M. E., Cutri R. M., Jarrett T., Kirkpatrick J. D., Padgett D., McMillan R. S., Skrutskie M., Stanford S. A., Cohen M., Walker R. G., Mather J. C., Leisawitz D., Gautier III T. N., McLean I., Benford D., Lonsdale C. J., Blain A., Mendez B., Irace W. R., Duval V., Liu F., Royer D., Heinrichsen I., Howard J., Shannon M., Kendall M., Walsh A. L., Larsen M., Cardon J. G., Schick S., Schwalm M., Abid M., Fabinsky B., Naes L., Tsai C.-W., 2010, AJ, 140, 1868

Yamagata T., Yoshii Y., 1992, AJ, 103, 117 